\title{
An incarcerated appendix and the ileocecum within a left inguinal hernia in an infant
}

\author{
Fumiya Yoneyama', Hideaki Tanaka ${ }^{1}$, Kentaro Ono ${ }^{1}$, Takato Sasaki ${ }^{1}$, Takahiro Jimbo $^{1}$, Chikashi Gotoh', \\ Toru Uesugi ${ }^{1}$, Yasuhisa Urita ${ }^{1}$, Toko Shinkai ${ }^{1}$, Hajime Takayasu', Natsuki Imoto ${ }^{2}$ and Kouji Masumoto ${ }^{1}$
}

\begin{abstract}
An 8-month-old boy with a left-sided incarcerated inguinal hernia involving the appendix, cecum, and terminal ileum was successfully managed via an inguinal approach during an emergency operation. A mobile cecum seemed to have contributed to the left-sided incarceration. Only 13 similar cases with the left-sided Amyand's hernia have been reported in the literature.
\end{abstract}

Keywords: Amyand's hernia; Left sided; Children; Inguinal hernia

\section{Background}

It has been reported in various series that the incidence of incarceration of an inguinal hernia in children ranges from 12 to $17 \%$ and is highest during the first year of life, decreasing thereafter [1]. The contents of the hernia sac may comprise the small bowel, cecum, appendix, omentum, or ovary and fallopian tube [2], and incarceration occurs more often on the right side (82 \%) [3].

We herein report a rare case of an incarcerated leftsided inguinal hernia involving the appendix, cecum, and terminal ileum.

\section{Case presentation}

An 8-month-old boy presenting with vomiting and swelling of the left inguino-scrotal region, which had been irreducible for 1 day, was referred to our department. The child had a history of a left inguinal hernia when he was 4 months old, which had been observed without surgical treatment. His vital signs were within the normal limits at this presentation. A physical examination revealed a tender, irreducible left-sided inguino-scrotal swelling. His abdomen was slightly distended with no tenderness. An ultrasonographic survey indicated that the hernia involved part of the intestines, the wall of which was quite edematous. The laboratory data were within normal limits,

\footnotetext{
* Correspondence: tanaka-h@md.tsukuba.ac.jp

'Department of Pediatric Surgery, Faculty of Medicine, University of Tsukuba, 1-1-1 Tennnoudai, Tsukuba, Ibaraki 305-8575, Japan

Full list of author information is available at the end of the article
}

except for the presence of anemia (hemoglobin $8.6 \mathrm{~g} / \mathrm{dl}$ ). An abdominal X-ray showed that there was no abnormal gas in the patient's left groin or scrotum but showed moderate dilation of the small bowel with gas. After unsuccessful manual reduction, we decided to perform an emergency operation for the incarcerated left-sided inguinal hernia.

After administration of general anesthesia, a transverse skin incision was performed on the left inguinal region. After opening the hernia sac, the appendix, terminal ileum, and cecum were found inside with slight congestion (Fig. 1). The intestines showed no perforation but had several serosal tears, which were repaired using absorbable sutures. Since the appendix was not inflamed, an appendectomy was not performed. After the contents of the hernia were reduced into the abdominal cavity, the hernia sac was dissected free from the spermatic cord, and a left herniorrhaphy was completed with high ligation of the sac (Potts procedure). The patient's postoperative course was uneventful. His chest-abdominal $\mathrm{X}$-ray did not indicate situs inversus in retrospect. A contrast study of the colon showed that his cecum was more centrally located than usual in the right lower quadrant, which was suggestive of a mobile cecum rather than of intestinal malrotation, judging from the running of the entire colon (Fig. 2). He was discharged in stable condition 2 days after the surgery. A recurrence of an inguinal hernia or testicular atrophy was not identified at his last follow-up visit 1 year postoperatively. 


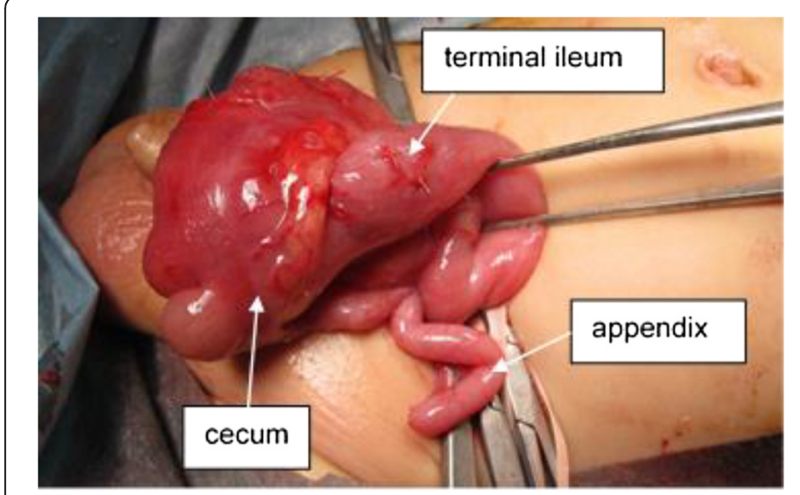

Fig. 1 The contents of an incarcerated left inguinal hernia exposed during an emergency operation: the appendix, cecum, and terminal ileum

\section{Discussion}

The presence of the appendix in an inguinal hernia sac is referred to as Amyand's hernia, named after Claudius Amyand, the first surgeon to describe and treat such a case in 1735, simultaneously performing the first appendectomy [4]. Since the hernia contents in our case included the appendix, our case can be categorized as a left-sided Amyand's hernia. The prevalence of Amyand's hernia is $0.4-0.6 \%$ among inguinal hernias in the general population and that in children is higher, reaching approximately $1 \%$ [4]. Because of its anatomical

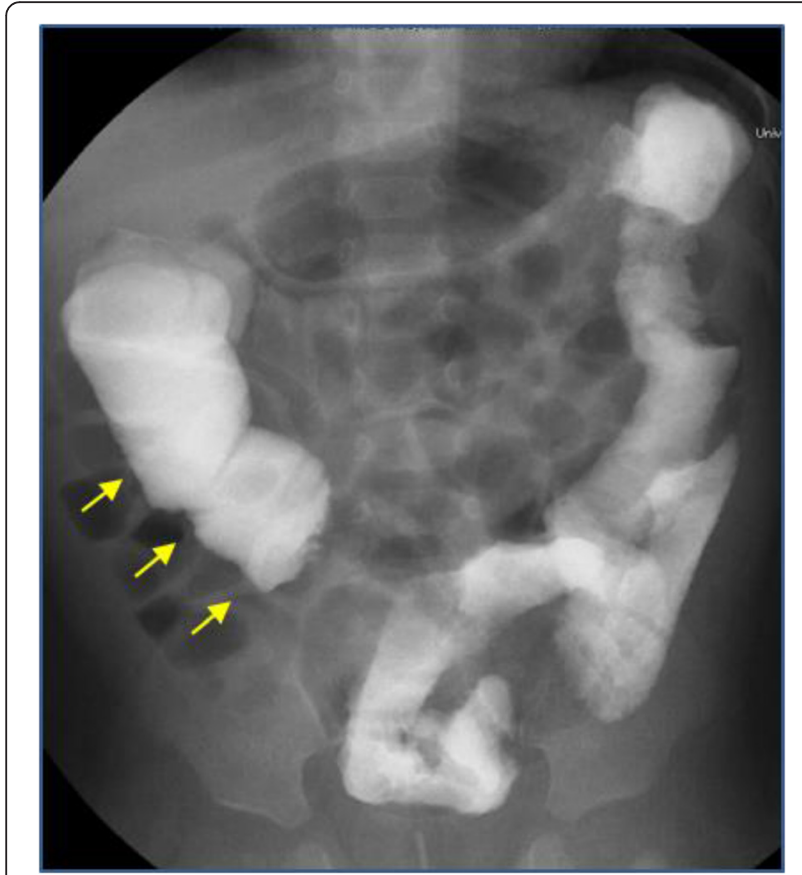

Fig. 2 A postoperative contrast study of the colon showed the cecum to be located in the right lower quadrant more centrally than usual (yellow arrows), indicating a mobile cecum position, the appendix is most commonly found in the right hernia sac and can be accompanied by the cecum and/or right colon. Amyand's hernia can appear on the left side rarely, and only 13 pediatric cases have been described in the literature [5-12].

Table 1 shows the demographics and clinical outcomes of the 14 pediatric cases, including ours, with left-sided Amyand's hernias. Some authors did not provide detailed information on their cases. To the best of the authors' knowledge, all of the patients were males whose ages ranged from 2 to 18 months. The presenting symptoms were mainly bilious or non-bilious vomiting, together with tender, irreducible left inguinal swelling. The contents other than the appendix included the cecum in eight cases, the sigmoid colon in one, and the terminal ileum in three. Perforation of the cecum was noted in one case, and serosal tear(s) of the terminal ileum and cecum were noted in each case, all of which were repaired during surgery. Normal appendices in six cases and four cases were preserved and resected, respectively. Inflamed or engorged appendices seen in four cases were all resected. All cases underwent routine herniorrhaphy and had excellent outcomes.

Left-sided Amyand's hernia is considered to involve associated situs inversus, intestinal malrotation, or a mobile cecum [5]. Among these three possible underlying conditions, 11 left-sided Amyand's hernias (Table 1) among the 14 cases showed the presence of a mobile cecum, and only 1 case had situs inversus as one of the main causes of the herniation, according to postoperative imaging studies. Regarding the preoperative imaging studies, ultrasonography was used in two cases including ours, but the actual contents could not be detected in either case (data not shown). Contrast-enhanced computed tomography (CT) and a contrast enema have revealed the presence of the ileocecum and/or appendix within left-sided Amyand's hernias in some adult cases [13-15]. These preoperative imaging studies would be useful for diagnosing Amyand's hernia and also the presence of situs inversus, intestinal malrotation, or a mobile cecum as possible underlying anomalies, especially in left-sided cases. However, it may not be practical to perform CT or a contrast enema preoperatively to rule out Amyand's hernia in all pediatric patients with symptoms of an incarcerated hernia, and physical examinations should be enough to prompt emergency operations in those patients.

Surgeons should be aware of and keep in mind the ileocecum as a content of an incarcerated hernia during surgery, even in the left inguinal region, because the ileocecum was included among the contents in almost all cases mentioned in our review. Appendicitis may rarely be encountered in Amyand's hernia, and the pathophysiology of appendiceal inflammation is considered to involve 
Table 1 The demographics and clinical outcomes of pediatric patients with left-sided Amyand's hernia, including our case

\begin{tabular}{|c|c|c|c|c|c|c|c|c|c|c|}
\hline Pt. No & Author & Year & Gender & Age & Presenting symptoms & Contents of hernia & $\begin{array}{l}\text { Presence of } \\
\text { appendicitis }\end{array}$ & Underlying condition & Surgical treatments & Outcome \\
\hline 1 & Gupta S [5] & 2005 & Male & 9 months & $\begin{array}{l}\text { Bilious vomiting, } \\
\text { irreducible inguinal } \\
\text { swelling }\end{array}$ & Appendix, cecum & $(-)$ & Mobile cecum & Herniorrhaphy & Uneventful \\
\hline 2 & Gupta N [6] & 2007 & Male & 11 months & $\begin{array}{l}\text { Bilious vomiting, } \\
\text { irreducible inguinal } \\
\text { swelling }\end{array}$ & $\begin{array}{l}\text { Appendix, cecum, } \\
\text { terminal ileum }\end{array}$ & $(+)$ & Mobile cecum & Herniorrhaphy, appendectomy & Uneventful \\
\hline 3 & Kaymakci A [7] & 2009 & N/A & N/A & N/A & N/A & \multirow{3}{*}{$\begin{array}{l}(+) \text { in one } \\
\text { of three }\end{array}$} & Mobile cecum & \multirow{3}{*}{$\begin{array}{l}\text { Herniorrhaphy in three, } \\
\text { appendectomy in one with } \\
\text { appendicitis }\end{array}$} & Uneventful \\
\hline 4 & Kaymakci A [7] & 2009 & $\mathrm{~N} / \mathrm{A}$ & $\mathrm{N} / \mathrm{A}$ & N/A & N/A & & Mobile cecum & & Uneventful \\
\hline 5 & Kaymakci A [7] & 2009 & $\mathrm{~N} / \mathrm{A}$ & $\mathrm{N} / \mathrm{A}$ & N/A & N/A & & Mobile cecum & & Uneventful \\
\hline 6 & Cankorkmaz L [8] & 2010 & Male & 4 months & $\begin{array}{l}\text { Vomiting, irreducible } \\
\text { inguinal swelling }\end{array}$ & Appendix & $(-)$ & \multirow{2}{*}{$\begin{array}{l}\text { Mobile cecum in } \\
\text { the one, not known } \\
\text { in the other }\end{array}$} & Herniorrhaphy, appendectomy & Uneventful \\
\hline 7 & Cankorkmaz L [8] & 2010 & Male & 2 months & $\begin{array}{l}\text { Irreducible inguinal } \\
\text { swelling }\end{array}$ & Appendix & $(-)$ & & Herniorrhaphy, appendectomy & Uneventful \\
\hline 8 & Khan R [9] & 2011 & Male & 10 months & Scrotum swelling & $\begin{array}{l}\text { Appendix, part of } \\
\text { the cecum }\end{array}$ & $(+)$ & Mobile cecum & $\begin{array}{l}\text { Herniorrhaphy, appendectomy, } \\
\text { another midline incision to } \\
\text { explore malrotation, cecopexy }\end{array}$ & $\begin{array}{l}\text { Uneventful except for } \\
\text { surgical site infection }\end{array}$ \\
\hline 9 & Singh K [10] & 2011 & Male & 18 months & $\begin{array}{l}\text { Bilious vomiting, fever, } \\
\text { irreducible inguinal } \\
\text { swelling }\end{array}$ & $\begin{array}{l}\text { Appendix, cecum } \\
\text { (perforation) }\end{array}$ & $(-)$ & Mobile cecum & $\begin{array}{l}\text { Herniorrhaphy, closure of cecal } \\
\text { perforation }\end{array}$ & Uneventful \\
\hline 10 & Singh K [10] & 2011 & Male & 18 months & Scrotum swelling & $\begin{array}{l}\text { Appendix, cecum, } \\
\text { terminal ileum } \\
\text { (serosal tear) }\end{array}$ & $(-)$ & Not known & $\begin{array}{l}\text { Herniorrhaphy, repair of serosal } \\
\text { tear }\end{array}$ & Uneventful \\
\hline 11 & Pun A [11] & 2013 & Male & 18 months & $\begin{array}{l}\text { Vomiting, irreducible } \\
\text { inguinal swelling }\end{array}$ & $\begin{array}{l}\text { Appendix, sigmoid } \\
\text { colon }\end{array}$ & $(+)$ & Mobile cecum & Herniorrhaphy, appendectomy & Uneventful \\
\hline 12 & Al-Mayoof [12] & 2014 & Male & 4 months & $\begin{array}{l}\text { Vomiting, irreducible } \\
\text { inguino-scrotal swelling }\end{array}$ & Appendix, cecum & $(-)$ & Situs inversus & Herniorrhaphy, appendectomy & Uneventful \\
\hline 13 & Al-Mayoof [12] & 2014 & Male & 10 months & $\begin{array}{l}\text { Bilious vomiting, fever, } \\
\text { irreducible inguinal } \\
\text { swelling }\end{array}$ & Appendix, cecum & $(-)$ & Mobile cecum & Herniorrhaphy, appendectomy & Uneventful \\
\hline 14 & Our case & 2014 & Male & 8 months & $\begin{array}{l}\text { Bilious vomiting, } \\
\text { irreducible inguinal } \\
\text { swelling }\end{array}$ & $\begin{array}{l}\text { Appendix, cecum } \\
\text { (serosal tears), } \\
\text { terminal ileum }\end{array}$ & $(-)$ & Mobile cecum & $\begin{array}{l}\text { Herniorrhaphy, repair of serosal } \\
\text { tears }\end{array}$ & Uneventful \\
\hline
\end{tabular}


ischemic events and trauma possibly due to incarceration, adhesion, and/or abnormal location of the appendix [16]. Appendicitis was noted in 4 of the 14 pediatric patients with left-sided Amyand's hernia (Table 1). The appendicitis patients were treated by appendectomy during an emergency operation. The other ten cases, including our own, had a normal appendix (six appendixes were left and untreated; four were resected). Although an appendectomy needs to be performed in the cases of Amyand's hernia with appendicitis, according to most reports, this procedure is not believed to be necessary when the appendix, as one of the hernia contents, shows no sign of inflammation. The reasons for hesitation in the resection of the normal appendix are as follows: (1) appendectomy may add to the risk of a surgical site infection [4]; (2) the incision may need to be enlarged to dissect the base of appendix, leading to the weakening of tissues and increasing the probability of a recurrence of inguinal hernia [17]; and (3) it may eliminate the possible use of the appendix in a later operation such as urinary diversion (Mitrofanoff appendicovesicostomy) [7].

One pediatric case with a left-sided Amyand's hernia underwent an intraoperative abdominal inspection for underlying anomalies via a midline incision [9], which indicated a mobile cecum. Recently, the laparoscopic approach has been demonstrated to offer an advantage over open procedures in patients with an incarcerated inguinal hernia, in that the laparoscopy can visualize the reduction and inspect the incarcerated organ [18]. However, the procedure is not yet commonly performed, and we did not choose the laparoscopic procedure for the treatment of our case because of our limited experience in performing laparoscopic surgery in emergency situations. Successful laparoscopic management of an adult right-sided Amyand's hernia was reported [19]. Therefore, laparoscopy would be more advantageous for leftsided hernias in children, because it can be used during surgery to confirm if the patient has the abovementioned underlying anomalies. Even if one of those anomalies is detected during a perioperative or intraoperative examination, no treatment is needed for mobile cecum or situs inversus. Intestinal malrotation, however, might require additional surgical intervention such as Ladd's procedure if the patient has a history of bowel obstruction that may have been caused by volvulus.

\section{Conclusions}

An 8-month-old boy with a left-sided incarcerated inguinal hernia, the contents of which involved a normal appendix, the cecum, and terminal ileum with serosal tears, was successfully managed using an inguinal approach during an emergency operation.

Similar left-sided Amyand's hernias in children have rarely been reported in the literature. A normal appendix can be preserved in such a situation, but an appendectomy and appropriate repair should be undertaken depending on the presence of infectious and/or traumatic changes among the hernia contents. Underlying anomalies such as a mobile cecum, intestinal malrotation, or situs inversus need to be investigated via imaging studies intra- or postoperatively.

\section{Consent}

Written informed consent was obtained from the patient's parents for publication of this case report and any accompanying images. A copy of the written consent is available for review by the Editor-in-Chief of this journal.

\section{Competing interests}

The authors declare that they have no competing interests.

\section{Authors' contribution}

FY and HIT participated in the patient's care, research design, performance of the research, and writing of the paper. HAT, TAS, and TJ participated in data analysis and writing the paper. $\mathrm{NI}$ and $\mathrm{KO}$ participated in the patient's care and data collection. CG, TU, YU, TOS, and KM participated in revising the manuscript critically. All authors read and approved the final manuscript.

\section{Authors' information}

Natsuki Imoto is a referring pediatrician. Kouji Masumoto is the professor; Fumiya Yoneyama, Takato Sasaki, and Kentaro Ono are trainees; Hideaki Tanaka and other authors are staff surgeons at the Department of Pediatric Surgery, University of Tsukuba.

\section{Author details}

${ }^{1}$ Department of Pediatric Surgery, Faculty of Medicine, University of Tsukuba, 1-1-1 Tennnoudai, Tsukuba, Ibaraki 305-8575, Japan. ²Department of Pediatrics, Ryugasaki Saiseikai Hospital, Ryugasaki, Ibaraki, Japan.

Received: 14 March 2015 Accepted: 22 July 2015

Published online: 31 July 2015

\section{References}

1. Grosfeld J, O'Neill J, Fonkals E, Coran A. Pediatric surgery 6th edn. St Louis, MO: Mosby/Elsevier; 2006. p. 1182.

2. Puri $P$, Hollwarth M. Pediatric surgery: diagnosis and management. Berlin, Heidelberg: Springer; 2006. p. 498

3. Rowe M, Clatworthy $\mathrm{H}$. Incarcerated and strangulated hernias in children: a statistical study of high risk factors. Arch Surg. 1970;101:136-7.

4. Michalinos A, Moris D, Vernadakis S. Amyand's hernia: a review. Am J Surg. 2014;207:989-95.

5. Gupta S, Sharma R, Kaushik R. Left-sided Amyand's hernia. Singapore Med J. 2005;46:424-5

6. Gupta N, Wilkinson T, Wilkinson A, Akhtar M. Left-sided incarcerated Amyand's hernia. Indian J Surg. 2007;69:17-8.

7. Kaymakci A, Akilliouglu I, Akkoyun I, Guven S, Ozdemir A, Gulen S. Amyand's hernia: a series of 30 cases in children. Hernia. 2009;13:609-12.

8. Cankorkmaz L, Ozer H, Guney C, Atalar M, Arslan M, Koyluoglu G. Amyand's hernia in the children: a single center experience. Surgery. 2010;147:140-3.

9. Khan R, Wahab S, Ghani I. Left-sided strangulated Amyand's hernia presenting as testicular torsion in an infant. Hernia. 2011;15:83-4.

10. Singh K, Singh R, Kaur S. Amyand's hernia. J Ind Asso Ped Surg. 2011;16:170-2

11. Pun A, Khatri R. Left sided Amyand's hernia with sliding component. J Nepal Med Assoc. 2013:52:285-7.

12. Al-Mayoof A, Al-Ani B. Left-sided Amyand hernia: report of two cases with review of literatures. Eur J Pediatr Surg Rep. 2014;2:63-6.

13. Bo D, Mojin W, Wei Z, Lie Y, Zongguang Z, Yinghan S. Successful management of an incarcerated left-sided Amyand's hernia in a 63-year-old male. Chin Med J. 2014;127:980-1 
14. Kinoo S, Aboobakar M, Singh B. Amyand's hernia: a serendipitous diagnosis. Case Rep Surg. 2013;2013:125095.

15. Unver M, Ozturk S, Karaman K, Turgut E. Left sided Amyand's hernia. World J Gastrointest Surg. 2013;5:285-6.

16. Kevorkian N, Rennie C, Asarian A, Pappas P. Left inguinal appendix in an HIV patient: a case report and review of the literature. Int I Surg Case Reports. 2013;4:293-5.

17. Psarras K, Lalountas M, Baltatzis M, Pavlidis E, Tsitlakidis A, Symeonidis N, et al. Amyand's hernia - a vermiform appendix presenting in an inguinal hernia: a case series. J Med Case Reports. 2011;5:463.

18. Esposite C, Turial S, Alicchio F, Enders J, Castagnetti M, Krause K, et al. Laparoscopic repair of incarcerated inguinal hernia. A safe and effective procedure to adopt in children. Hernia. 2013;17:235-9.

19. Vermillion J, Abernathy S, Snyder S. Laparoscopic reduction of Amyand's hernia. Hernia. 1999;3:159-60.

Submit your manuscript to a SpringerOpen ${ }^{\circ}$ journal and benefit from:

- Convenient online submission

- Rigorous peer review

- Immediate publication on acceptance

- Open access: articles freely available online

- High visibility within the field

- Retaining the copyright to your article

Submit your next manuscript at $\boldsymbol{\nabla}$ springeropen.com 